\title{
Factores asociados a la satisfacción del paciente en diálisis
}

\author{
Ana Belén Romero-Ruiz, Alfonso Sevillano-Jiménez y Cristina Cardador-Trocoli \\ Departamento de Enfermería. Facultad de Medicina y Enfermería. Universidad de Córdoba. España
}

Como citar este artículo: Romero-Ruiz AB, Sevillano-Jiménez A, Cardador-Trócoli C. Factores asociados a la satisfacción del paciente en diálisis. Enferm Nefrol. 2019 Abr-Jun;22(2):112-23

\section{Resumen}

Introducción: La satisfacción del paciente es uno de los grandes retos que persigue conseguir el sistema sanitario y que repercute de forma positiva en la esfera biopsicosocial del paciente.

Constituye una prioridad de los profesionales sanitarios el velar por dicha satisfacción y ello pasa por conocer las necesidades del paciente para luego marcar objetivos que el personal debe cumplir.

Objetivo: Analizar la satisfacción del paciente en diálisis a partir de la producción científica existente.

Material y Método: Se ha realizado una revisión integrativa mediante una búsqueda en las bases de datos Pubmed, Cuiden, Google académico y Medes. Se incluyeron artículos científicos originales y de revisión, en inglés y español, excluyendo aquellos artículos que no presentaban resultados o que no estuviesen en texto completo.

Resultados: Se revisaron 17 artículos que se ajustaron a los criterios de selección. Los principales resultados encontrados se agruparon en los siguientes aspectos: factores determinantes en el nivel de satisfacción del paciente, herramientas de valoración de la satisfacción, rol de enfermería y áreas de mejora.

Conclusiones: Los factores más influyentes en la satisfacción del paciente en diálisis son, principalmente, la confianza transmitida y disposición del personal sanitario, trato personalizado, amabilidad y seguridad trans-

Correspondencia:
Ana Belén Romero Ruiz
C/ General Lázaro Cárdenas, N³ (Manz 5), Portal 3, 2³
14013 Córdoba
E-mail: anabelen_rr_93@hotmail.com

mitida, la destreza para puncionar, entre otros. Respecto a los instrumentos de medida, no existe un instrumento de medida específico, para evaluar la satisfacción del paciente en diálisis. Los atributos más satisfactorios que destacan en la enfermería, son amabilidad, disposición e interés, preparación técnica profesional, trato personalizado, confianza y seguridad clínica.

PALABRAS CLAVE: diálisis; satisfacción del paciente; factores.

\section{Factors associated with satisfaction in dialysis patients}

\section{Abstract}

Aims: Patient satisfaction is one of the great challenges that the healthcare system seeks to achieve and that has a positive impact on the biopsychosocial sphere of the patient.

It is a priority for healthcare professionals to ensure this satisfaction and this involves knowing the needs of the patient and then setting targets that the staff must meet.

Objective: To analyse the dialysis patient satisfaction according to existing scientific production.

Material and Method: An integrative review has been carried out through a search of PubMed, Cuiden, Google academic and Medes databases. Original and review articles were included, in English and Spanish, excluding those articles that did not present results or that were not in full text.

Results: We reviewed 17 articles that met the selection criteria. The main results found were grouped into 
the following aspects: determining factors in the level of patient satisfaction, satisfaction assessment tools, nursing role and areas for improvement.

Conclusions: The most influential factors in the satisfaction of dialysis patient are, mainly, the transmitted confidence and disposition of the health personnel, personalized treatment, kindness and transmitted security, the skill to puncture, among others. Regarding measurement instruments, there is no specific measurement instrument to evaluate satisfaction in dialysis patient. The most satisfactory attributes that stand out in nursing are kindness, willingness and interest, professional technical preparation, personalized treatment, confidence and clinical safety.

KEYWORDS: dialysis; patient satisfaction; factors.

\section{Introducción}

El aumento de la esperanza de vida viene determinado por un cambio en los estilos de vida y avances en el ámbito de la sanidad. Como consecuencia de esto, nos encontramos con una población cada vez más envejecida; lo que conlleva a un mayor número de problemas de salud y un aumento del uso de los recursos sanitarios ${ }^{1-3}$.

La Enfermedad Renal Crónica Avanzada (ERCA) es, hoy por hoy, un problema de salud pública en todo el mundo por su prevalencia e incidencia creciente en la población, su naturaleza crónica y permanente, su impacto en el paciente, su familia y el sistema sanitario $0^{4,5}$.

Como principal medida para tratar la ERCA encontramos el trasplante renal, el cual representa actualmente el tratamiento más eficaz para tratar a los pacientes con ERCA, pero la escasez de donantes lo convierte en una posibilidad no disponible para todos los pacientes; haciendo que la mayoría de ellos tengan que tratarse con otra terapia renal sustitutiva (TRS) como la hemodiálisis (HD) o la diálisis peritoneal (DP) y deban acostumbrarse a las monitores, punciones de la fístula y catéteres necesarios para llevar a cabo los procedimientos que los mantengan vivos ${ }^{5}$.

Estos procedimientos ocasionan grandes cambios en la vida del enfermo, tanto a nivel físico, psicológico como social; por lo que se hace necesario un esfuerzo por parte de los servicios de salud para ofrecer cuidados y atención de calidad ${ }^{1,5}$.
El esfuerzo de los profesionales de enfermería se ha centrado siempre en prestar cuidados de calidad. Es por ello, que en lo últimos años se vuelven relevantes aspectos como la calidad de vida, la salud percibida y el bienestar del paciente y su familia. Es por esto que el paciente se convierte en el centro de la asistencia sanitaria, siendo sus necesidades las directrices que se debe marcar el sistema sanitario para ofrecer las prestaciones necesarias para ayudar a los ciudadanos ${ }^{6}$.

La importancia de satisfacer estas necesidades radica en que al mejorar la perspectiva del paciente respecto a la enfermedad, estamos ayudando a afrontarla, lo que influye positivamente en el proceso de esta ${ }^{7}$.

Esta reflexión, nos lleva de nuevo a percibir la importancia de los aspectos relacionados con el bienestar del enfermo. Sin embargo, la calidad de un servicio es un término complicado de definir, ya que va a depender de las características de cada paciente en concreto y se rige por las diferencias en los estilos de vida, experiencias de salud percibidas anteriormente y características sociales de cada persona ${ }^{6,7}$.

El comienzo del ciclo para conseguir la satisfacción del paciente sería conocer las necesidades y expectativas de éste con el sistema sanitario, para luego poder establecer objetivos a cumplir por el personal sanitario $0^{2,7}$.

Para conocer estas necesidades, necesitamos ayudarnos de instrumentos. Uno de ellos es el cuestionario SERVQUOS. Se han llevado a cabo múltiples estudios en los que se ha utilizado dicha herramienta en pacientes en diálisis y se han conseguido conocer algunos de los aspectos de la atención sanitaria que más satisfacen a los pacientes; elementos como la puntualidad en las sesiones de HD o el interés del personal de enfermería por los pacientes, son los hechos que más satisfacción brindan 2,3,6.

En este sentido, el análisis de la satisfacción del paciente es muy útil para diseñar estrategias de mejora en la asistencia y modificar ciertos aspectos para lograr prestar cuidados de calidad, siendo estos imprescindibles para la seguridad del paciente. Dentro de estas oportunidades de mejora, podemos citar aquellos factores que aumentan la confianza del paciente en el personal sanitario como son: el tiempo dedicado a la consulta o el conocimiento del nombre del profesional por parte del paciente ${ }^{2,3}$.

El personal de enfermería es el que mantiene el primer contacto con el paciente en diálisis; y por ello el responsable de crear una buena relación enfermero-paciente, 
lo que ayudará a crear un ambiente de confianza, credibilidad y comunicación entre el paciente, la familia y el personal sanitario; todo ello será más factible si añadimos una atención de calidad y cubrimos las necesidades de la persona a la que estamos atendiendo ${ }^{8}$.

Por todo ello, y dada la relevancia que presenta la satisfacción del paciente en tratamiento con diálisis, nos planteamos como objetivo principal revisar la bibliografía científica existente sobre la satisfacción del paciente en diálisis, con los siguientes objetivos secundarios:

- Conocer los factores más influyentes en la satisfacción del paciente en diálisis.

- Precisar los instrumentos de medida más utilizados en la valoración de la satisfacción del paciente en diálisis.

- Determinar los aspectos más destacados de la satisfacción por parte de los pacientes en diálisis de su interacción con enfermería.

\section{Metodología}

Se llevó a cabo una revisión integrativa de la literatura9. Este tipo de revisión presenta un enfoque más sistemático y riguroso que la revisión narrativa de la bibliografía tradicional, estableciendo la consecución de criterios que aseguran el nivel de calidad deseado de los resultados obtenidos.

El desarrollo de la revisión integrativa del presente estudio lleva consigo el establecimiento de diferentes fases bien definidas, destacando:

\section{Identificación del objetivo de la revisión:}

¿Cuáles son los factores determinantes en la satisfacción del paciente en tratamiento con diálisis?

\section{Búsqueda y selección de artículos:}

La búsqueda de la bibliografía seleccionada se realizó a través diversas bases de datos con el objetivo de recopilar la mayor producción científica posible disponible en relación con el objeto a estudio. Como determinante, se estableció la selección de artículos publicados en los últimos 5 años (periodo comprendido entre 2013 y 2018). El periodo de búsqueda bibliográfica fue establecido desde Marzo hasta Junio de 2018. Las bases de datos empleadas fueron: Pubmed, Cuiden, Google académico y Medes.
El método de búsqueda consistió en utilizar como palabras clave o descriptores: diálisis (dialysis), satisfacción (satisfaction), paciente (patient), factores (factors). En cada base de datos se llevó a cabo estrategias de búsqueda diferentes, llevando consigo diversas pruebas de asociación entre los descriptores anteriormente citados mediante operadores boleanos ("AND", "OR"), teniendo presente la existencia de cada uno de los descriptores empleados en: resumen, título del artículo y/o palabras clave. La metodología de búsqueda empleada fue determinada y condicionada a los siguientes criterios:

\section{a) Criterios de Inclusión:}

- Artículos en español e inglés.

- Artículos relacionados con el tema de interés.

- Artículos originales y de revisión a texto completo.

\section{b) Criterios de Exclusión:}

- Documentos provenientes de bases de datos sin evidencia científica ni fiabilidad derivada.

- Artículos que no presentasen los criterios de inclusión anteriormente mencionados.

\section{Lectura crítica:}

Al inicio de la búsqueda no se consideró ningún criterio de inclusión/exclusión en las diversas bases de datos, con el objetivo de conocer la cantidad de producción científica existente respeto al tema a tratar, obteniendo, de este modo, mayor conocimiento del mismo, aceptándose todo aquel artículo que tratase sobre el tema en cuestión.

Tras la lectura de títulos y resúmenes de todos los artículos potencialmente seleccionables, se descartaron aquellos que no cumpliesen con los criterios de inclusión y exclusión establecidos. Posteriormente, se realizó la lectura completa de los trabajos seleccionados, realizándose un nuevo cribado de aquellos que no versaban sobre el objeto a estudio.

Finalizado dicho proceso de selección, el número de artículos total que cumplían con los criterios de inclusión marcados y que se han utilizado para el estudio han sido de 17 (Tabla 1). En ese momento se comenzó el análisis de los artículos recopilados.

Tabla 1. Procedencia de los artículos seleccionados.

\begin{tabular}{|ccccc}
\hline Bases de Datos & PUBMED & CUIDEN & $\begin{array}{c}\text { GOOGLE } \\
\text { SCHOLAR }\end{array}$ & MEDES \\
\hline $\begin{array}{c}\text { Total de publicaciones } \\
\text { localizadas }\end{array}$ & 109 & 34 & 142 & 11 \\
$\begin{array}{c}\text { Total de publicaciones } \\
\text { seleccionadas }\end{array}$ & 4 & 5 & 6 & 2 \\
\hline
\end{tabular}


Integración de los hallazgos:

La información analizada dentro de los artículos obtenidos fue: definición e identificación de factores determinantes en la satisfacción implícita y explícita de los pacientes sometidos al proceso de diálisis, recopilación de principales herramientas de valoración a cerca de la satisfacción y rol de enfermería respecto al desarrollo de la mencionada variable.

Tras ello, se procedió a la interpretación de la información recopilada, así como el establecimiento de las conclusiones obtenidas.

\section{Resultados}

\section{Selección de estudios preliminar:}

De un total de 296 artículos preseleccionados, se excluyeron 244 durante la revisión de títulos y resúmenes. Otros 35 fueron eliminados tras la revisión del texto completo. Finalmente, se seleccionaron 17 artículos que cumplían con los criterios de inclusión/exclusión previamente establecidos, tal como se refleja en la Figura 1.

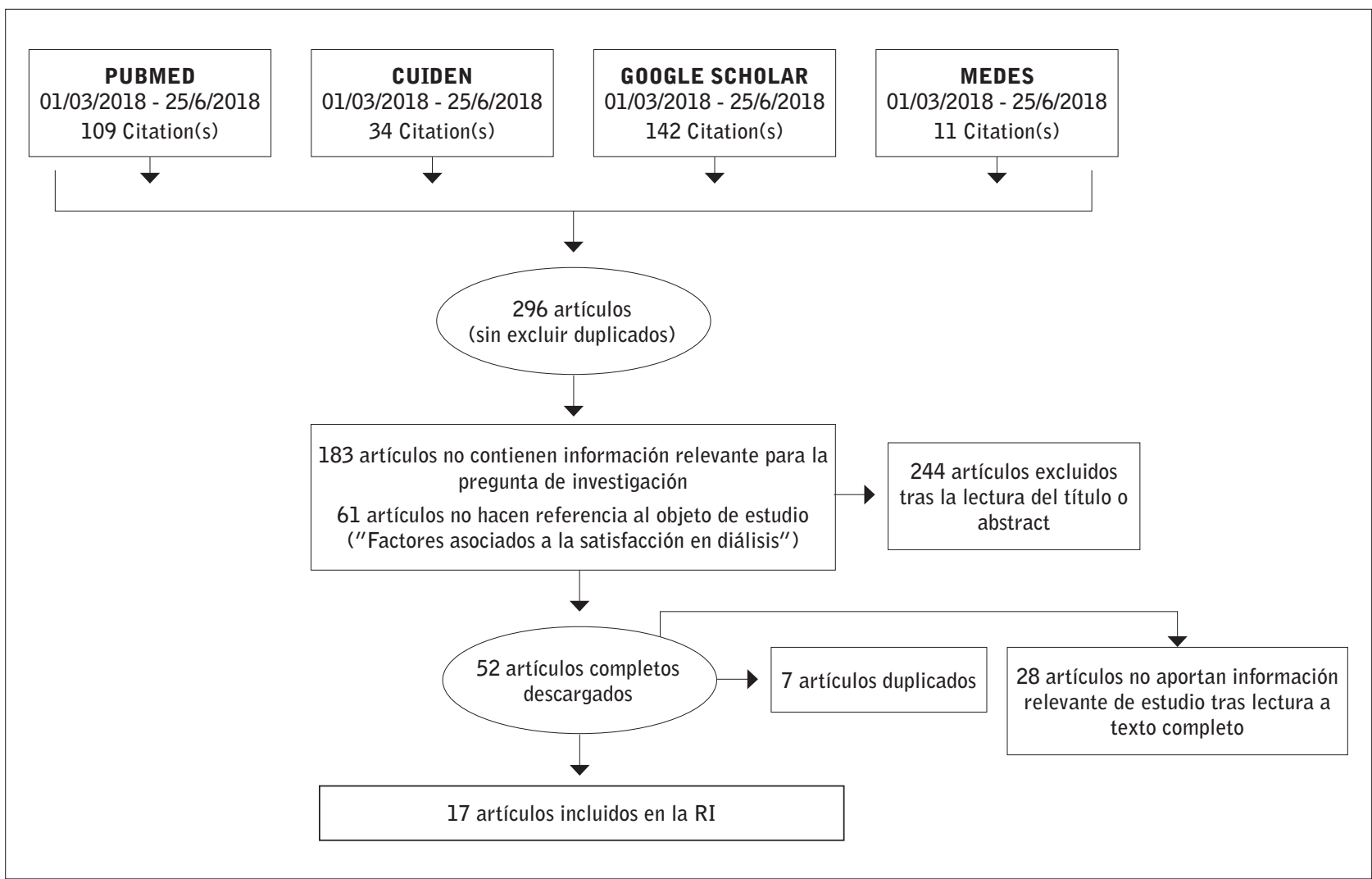

Figura 1. Diagrama de flujo de las etapas metodológicas utilizadas en la Revisión Integrativa de la Literatura. 


\section{Características de los estudios}

\section{Los datos más relevantes de cada estudio se muestran en la Tabla 2.}

Tabla 2. Artículos revisados.

\begin{tabular}{|c|c|c|c|}
\hline Autor, Año, País & Diseño del estudio & Muestra & Principales conclusiones \\
\hline $\begin{array}{c}\text { García R, et al. } 2016 . \\
\text { España3 }\end{array}$ & $\begin{array}{l}\text { Estudios de corte } \\
\text { transversal. }\end{array}$ & $\begin{array}{c}143 \\
\text { participantes. }\end{array}$ & $\begin{array}{l}\text { Un elevado porcentaje indica que los pacientes colaboran } \\
\text { de buen grado. Los pacientes en diálisis se muestran } \\
\text { satisfechos pero debemos mejorar en la información } \\
\text { relacionada con la medicación. Este es el punto que nos } \\
\text { plantemos como objetivo de mejora. }\end{array}$ \\
\hline $\begin{array}{c}\text { Sanz M, et al. } 2017 \\
\text { España }{ }^{7} .\end{array}$ & $\begin{array}{l}\text { Estudio cualitativo de tipo } \\
\text { fenomenológico, mediante } \\
\text { grupo focal. }\end{array}$ & 7 participantes. & $\begin{array}{l}\text { Los pacientes en diálisis destacan la relación que se crea } \\
\text { entre ellos y enfermería. Tienen un alto grado de confianza } \\
\text { en el personal de enfermería, basado en su destreza para } \\
\text { pinchar la fístula arteriovenosa y en su fiabilidad pro- } \\
\text { fesional. }\end{array}$ \\
\hline $\begin{array}{l}\text { Rodríguez D. } 2013 \\
\text { España10. }\end{array}$ & $\begin{array}{l}\text { Estudio descriptivo y } \\
\text { correlacional. }\end{array}$ & 54 participantes. & $\begin{array}{l}\text { El índice de satisfacción fue de } 86,36 \% \text { para el ítem } \\
\text { referido a la satisfacción general, teniendo una valoración } \\
\text { de excelente. Los } 5 \text { ítems más valorados fueron: la } \\
\text { confianza trasmitida, la disposición del personal, el trato } \\
\text { personalizado, la amabilidad del personal y la información } \\
\text { relacionada con la higiene. Los menos valorados fueron: } \\
\text { la señalización para llegar al Servicio, la capacitación del } \\
\text { personal, la información médica, la tecnología de los equipos } \\
\text { y el tiempo de espera para ser atendido por el médico. }\end{array}$ \\
\hline $\begin{array}{c}\text { Martín RA. } 2014 . \\
\text { México }{ }^{11} .\end{array}$ & $\begin{array}{l}\text { Estudio observacional, } \\
\text { descriptivo y trasversal. }\end{array}$ & 70 participantes. & $\begin{array}{l}\text { El análisis de la satisfacción del paciente es muy útil para } \\
\text { diseñar una estrategia de mejora en la asistencia enfermera } \\
\text { y prestar cuidados de calidad. Los cuidados de calidad son } \\
\text { determinantes para la seguridad del paciente. }\end{array}$ \\
\hline $\begin{array}{c}\text { Gómez M, et al.2014 } \\
\text { España }{ }^{12} \text {. }\end{array}$ & $\begin{array}{l}\text { Estudio observacional, } \\
\text { descriptivo y trasversal. }\end{array}$ & 70 participantes. & $\begin{array}{l}\text { La mayoría de los pacientes evalúan positivamente el } \\
\text { trabajo de la enfermera, excepto en lo referente a la } \\
\text { información sobre dieta y medicación, que se muestran } \\
\text { más insatisfechos. Por tanto será en este sentido en el que } \\
\text { debamos esforzarnos más. No se observó correlación entre } \\
\text { variables socio-demográficas y el grado de satisfacción. } \\
\text { El análisis de la satisfacción del paciente es muy útil para } \\
\text { diseñar una estrategia de mejora en la asistencia enfermera } \\
\text { y prestar cuidados de calidad. }\end{array}$ \\
\hline $\begin{array}{l}\text { López MC, et al. } 2016 . \\
\qquad \text { España }{ }^{13} \text {. }\end{array}$ & $\begin{array}{l}\text { Estudio observacional, } \\
\text { descriptivo y transversal. }\end{array}$ & 89 participantes. & $\begin{array}{c}\text { Los pacientes que reciben tratamiento de diálisis en nuestra } \\
\text { UGC, se reconocen muy satisfechos con ligeras diferencias } \\
\text { según la unidad en la reciben tratamiento, y conocer } \\
\text { aspectos mejorables en el servicio, que se están abordando } \\
\text { para aumentar la satisfacción del paciente y por tanto la } \\
\text { calidad asistencial. }\end{array}$ \\
\hline $\begin{array}{l}\text { Rebollo A et al. } 2015 \\
\text { España } \\
\text { Es. }\end{array}$ & $\begin{array}{l}\text { Estudio observacional, } \\
\text { descriptivo y transversal. }\end{array}$ & 83 participantes. & $\begin{array}{l}\text { Aspectos tales como la calidad de vida, la satisfacción del } \\
\text { enfermo y el nivel de salud percibida por éste, han cobrado } \\
\text { especial importancia en los últimos años en el tratamiento } \\
\text { de diálisis. Este estudio estudió las variables anteriores, } \\
\text { dando como resultado un alto grado de satisfacción en } \\
\text { pacientes que iniciaban la terapia renal sustitutiva. }\end{array}$ \\
\hline
\end{tabular}




\begin{tabular}{|c|c|c|c|}
\hline Autor, Año, País & Diseño del estudio & Muestra & Principales conclusiones \\
\hline $\begin{array}{c}\text { Aguilar F et al. } 2015 \\
\text { Brasil }^{15} .\end{array}$ & $\begin{array}{l}\text { Estudio observacional, } \\
\text { descriptivo y transversal. }\end{array}$ & $\begin{array}{c}338 \\
\text { participantes. }\end{array}$ & $\begin{array}{l}\text { La diálisis peritoneal fue más favorable con respecto a } \\
\text { la calidad de vida ( } p<0,05 \text { ), debido a la gran cantidad de } \\
\text { elementos con resultados significativos en comparación con } \\
\text { la Hemodiálisis. Sin embargo, las dos variables de mayor } \\
\text { importancia encontradas en la hemodiálisis (funcionamiento } \\
\text { físico - } p<0,05 \text { y funcionamiento emocional } p<0,01 \text { ) } \\
\text { terminaron teniendo un impacto mucho mayor en el } \\
\text { bienestar y la vida diaria del paciente en el entorno externo } \\
\text { a la clínica que aquellos que eran más altos en diálisis } \\
\text { peritoneal, haciendo de la hemodiálisis la más favorable } \\
\text { para la calidad de vida del paciente. }\end{array}$ \\
\hline $\begin{array}{c}\text { Sanabria M et al. } 2017 \\
\text { Colombia }{ }^{16} .\end{array}$ & $\begin{array}{l}\text { Estudio observacional, } \\
\text { descriptivo y transversal. }\end{array}$ & $\begin{array}{l}370 \\
\text { participantes. }\end{array}$ & $\begin{array}{l}\text { La escala ESUR-HD mide la satisfacción de los pacientes } \\
\text { en hemodiálisis en una dimensión con } 9 \text { dominios. EI } \\
\text { instrumento puede detectar cambios en el constructo, } \\
\text { gracias a la adecuada validez y fiabilidad de la misma. Las } \\
\text { versiones subsiguientes de la escala deben incluir nuevos } \\
\text { elementos que permitan una mejor discriminación entre los } \\
\text { altos niveles de satisfacción. }\end{array}$ \\
\hline $\begin{array}{l}\text { Ángel ZE et al. } 2016 \\
\text { Colombia }\end{array}$ & Revisión Sistemática. & 21 artículos. & $\begin{array}{l}\text { Materias como los modelos y teorías de enfermería en el } \\
\text { cuidado del paciente en hemodiálisis, el uso de lenguaje } \\
\text { común por parte de enfermería, los diagnósticos de } \\
\text { enfermería, la educación y autocuidado del paciente y la } \\
\text { implicación de la familia como factor importante en la vida } \\
\text { del enfermo; son las que hacen que la atención al paciente } \\
\text { en hemodiálisis roce la excelencia. }\end{array}$ \\
\hline $\begin{array}{c}\text { Rocha MR et al. } 2014 \\
\text { México }^{18} \text {. }\end{array}$ & $\begin{array}{l}\text { Estudio descriptivo } \\
\text { longitudinal. }\end{array}$ & $\begin{array}{l}100 \% \text { pacientes } \\
\text { ingresados } \\
\text { en urgencias, } \\
\text { hemodiálisis y } \\
\text { hospitalización. }\end{array}$ & $\begin{array}{l}\text { La calidad de la atención se contempla como indicador de } \\
\text { la satisfacción de los pacientes. En este estudio se demostró } \\
\text { que la calidez del trato, el tiempo de espera y la información } \\
\text { que se proporciona ante cualquier actuación que se realice, } \\
\text { son factores que determinan la satisfacción del paciente. }\end{array}$ \\
\hline $\begin{array}{l}\text { Urbina MA et al. } 2016 \\
\text { México }^{19} \text {. }\end{array}$ & $\begin{array}{l}\text { Estudio observacional, } \\
\text { descriptivo y transversal. }\end{array}$ & $\begin{array}{c}178 \\
\text { participantes. }\end{array}$ & $\begin{array}{c}\text { Prestar servicios de alta calidad a los pacientes y brindar } \\
\text { calidad en el cuidado de la salud, es la razón de ser de } \\
\text { la formación de enfermería. En este estudio, se observó } \\
\text { que la implementación del cuidado en las áreas de diálisis } \\
\text { peritoneal y hemodiálisis resultan satisfactorias para el } \\
\text { paciente, aunque aún quedan amplias zonas para estudiar } \\
\text { mejoras. }\end{array}$ \\
\hline $\begin{array}{c}\text { Morga S et al. } 2014 \\
\text { España }\end{array}$ & Estudio descriptivo. & 31 participantes. & $\begin{array}{l}\text { Se midió el grado de satisfacción de los pacientes por medio } \\
\text { de tres cuestionarios (SF-36, LOPSS } 12 \text { y un cuestionario } \\
\text { de opinión de los cuidados de enfermería dirigido a los } \\
\text { enfermos del centro Fresenius Medical Care E palmar } \\
\text { (Murcia), dando como resultados de este último una óptima } \\
\text { calidad de vida de los pacientes y un afrontamiento positivo } \\
\text { de éstos ante la enfermedad. }\end{array}$ \\
\hline $\begin{array}{c}\text { Palmer SC et al. } 2014 \\
\text { Italia }{ }^{21} \text {. }\end{array}$ & $\begin{array}{l}\text { Estudio observacional, } \\
\text { descriptivo y transversal. }\end{array}$ & $\begin{array}{l}2748 \\
\text { participantes. }\end{array}$ & $\begin{array}{l}\text { Los pacientes sometidos a hemodiálisis están menos } \\
\text { satisfechos con los aspectos complejos de la atención. Las } \\
\text { expectativas de los pacientes respecto a la información } \\
\text { respecto al pronóstico, la probabilidad de trasplante de } \\
\text { riñón y sus opciones al elegir el tratamiento de diálisis } \\
\text { deben considerarse al planificar la investigación y las } \\
\text { prácticas de atención médica derivadas. }\end{array}$ \\
\hline $\begin{array}{l}\text { Huertas MP et al. } \\
\text { 2014. España }{ }^{22} \text {. }\end{array}$ & $\begin{array}{l}\text { Estudio observacional, } \\
\text { descriptivo y transversal. }\end{array}$ & 35 participantes. & $\begin{array}{l}\text { Es de suma importancia tratar el factor psicológico de los } \\
\text { pacientes en hemodiálisis crónica, pues en este estudio } \\
\text { se comprueba que variables como el índice de depresión } \\
\text { afectan significativamente tanto a la adherencia al } \\
\text { tratamiento como al bienestar general del enfermo renal. }\end{array}$ \\
\hline
\end{tabular}




\begin{tabular}{|c|c|c|c|}
\hline Autor, Año, País & Diseño del estudio & Muestra & Principales conclusiones \\
\hline $\begin{array}{c}\text { Reid C et al. } 2016 \\
\text { EE.UU. }{ }^{23} \text {. }\end{array}$ & $\begin{array}{l}\text { Revisión bibliográfica de } \\
\text { la literatura. }\end{array}$ & $\begin{array}{l}17 \text { estudios (576 } \\
\text { participantes). }\end{array}$ & $\begin{array}{c}\text { Centrarse en las intervenciones que se incorporan a las } \\
\text { relaciones establecidas que los pacientes tienen con sus } \\
\text { profesionales de la salud puede permitirles progresar } \\
\text { hacia un sentido de control y mejorar la satisfacción con la } \\
\text { atención. Se considera que estas relaciones influyen través } \\
\text { del intercambio de información, la continuidad y el soporte } \\
\text { personalizado. }\end{array}$ \\
\hline $\begin{array}{l}\text { Ruiz de Alegría B et al. } \\
2013 \text { España }{ }^{24} \text {. }\end{array}$ & Estudio longitudinal. & 98 participantes. & $\begin{array}{c}\text { Ante la visión negativa que supone el inicio del tratamiento } \\
\text { de diálisis, se torna imprescindible identificar los factores } \\
\text { que afectan a la calidad de vida de los pacientes y a su } \\
\text { bienestar psicológico. En este estudio se logra ver como } \\
\text { las estrategias de evitación y aproximación son las, más } \\
\text { influyentes en el bienestar psicológico de los pacientes en } \\
\text { diálisis, por ello es que los profesionales deben de conocer } \\
\text { cómo detectarlas precozmente. }\end{array}$ \\
\hline
\end{tabular}

\section{Descripción de los resultados en función de las variables}

\section{Factores determinantes en el nivel de satisfacción del paciente}

La mayoría de artículos seleccionados evidencian que los principales factores determinantes de una mayor satisfacción del paciente en diálisis son, principalmente, la confianza transmitida ${ }^{10,11}$ y la disposición del personal sanitario en la prestación de ayuda ${ }^{10-12}$, el trato personalizado ${ }^{10-13}$, amabilidad y seguridad transmitida $7,10,11,13$, la destreza para pinchar la fístula ${ }^{7}$, interés por resolución de problemas asociados $3,11,13$, así como la información relacionada con la higiene y la rapidez de respuesta requerida ${ }^{10}$.

Por otro lado, los ítems peor valorados por los pacientes, según la bibliografía revisada, serían: señalización para llegar al servicio ${ }^{10}$, capacitación del personal ${ }^{10}$, información médica ${ }^{3,10}$, tecnología de los equipos, $3,10,13$, tiempo de espera de atención médica ${ }^{7,10,13}$, así como la información referente a la dieta y medicación prescri$\mathrm{ta}^{3,11,12}$, destacando éste último como el ítem en el que los pacientes reportan mayor insatisfacción. Asimismo, $\operatorname{varios}^{7,13}$, coinciden en que la falta de personal en las unidades de diálisis sería un factor determinante en el incremento de insatisfacción de los pacientes.

Algunos estudios apuntan leves diferencias entre la satisfacción percibida por los pacientes de hemodiálisis con respecto a la diálisis peritoneal, siendo comparativamente mejores en esta última ${ }^{3,14}$.
Cabe destacar, la valoración general positiva de los pacientes respecto al trabajo desempeñado por los profesionales de Enfermería en la unidad de diálisis, aspecto que deriva en un aumento de calidad percibida hacia dicha unidad $3,11-13$.

\section{Herramientas de valoración de la satisfacción}

Según diversos autores, el análisis de la satisfacción del paciente nos permite diseñar una estrategia de mejora en la atención enfermera y prestación de cuidados de calidad, ya que las necesidades del paciente constituyen el eje sobre el que se articulan las prestaciones asistenciales ${ }^{10,16,17}$. Para ello, se precisa la utilización de herramientas de valoración que nos permitan conocer el estado actual de satisfacción del paciente en diversas áreas de estudio, según los diferentes factores que la conforman ${ }^{7,14}$.

Sin embargo, no existe un instrumento de medida específico, para evaluar la satisfacción del paciente en diálisis; y los existentes no están adaptados a la población española en diálisis.

Atendiendo a la selección de artículos del presente trabajo, existen diferentes instrumentos, que permiten conocer de diferentes formas el grado de satisfacción del paciente en diálisis. En este sentido, algunos cuestionarios diseñados para medir la calidad, añaden como variable la satisfacción ${ }^{18}$ y los cuestionarios de valoración CARE-Q (escala análoga de 4 puntos) ${ }^{19}$ y LOPSS 12 para evaluar el nivel de satisfacción de los cuidados enfermeros ${ }^{20}$. 
En efecto, uno de los factores que intervienen en la calidad de vida de los pacientes en diálisis, es la satisfacción. Así encontramos el cuestionario KDQOL-SF (Kidney Disease Quality of Life - Short Form), el cual está indicado específicamente para evaluar el grado de calidad de vida relacionada con la salud en los pacientes con afectación renal ${ }^{14}$. Este cuestionario, asociado con el ya mencionado SF-36, conforman el integrado KDQOL SF-36, permitiendo, conocer el nivel de satisfacción de los pacientes nefrológicos, tanto en HD como en DP14,15.

Otro de los instrumentos utilizados es el SERVQHOS ${ }^{10,11}$, que evalúa la calidad percibida por el paciente, aplicada en estudios de satisfacción de los usuarios, combinando expectativas y percepciones ${ }^{10}$. Además de discriminar entre pacientes satisfechos e insatisfechos así como, permitirnos identificar oportunidades de mejora $3,7,10-13$. Este cuestionario ha demostrado una alta consistencia interna, una buena capacidad predictiva, y especialmente una buena estructura factorial $^{10,12}$.

Del mismo modo, resulta evidente resaltar el cuestionario CHOICE (Cuestionario de elecciones para resultados saludables en el cuidado de la enfermedad renal en etapa terminal), que evalúa la satisfacción el paciente con el tratamiento llevado a cabo en diálisis ${ }^{21}$. Dicha herramienta consta de 20 preguntas sobre las experiencias de los pacientes en relación con los diferentes aspectos de la atención de diálisis. Además, tres preguntas le piden a los pacientes que califiquen la calidad general de su atención, cuánto de su atención podría ser mejor y si recomendarían el Servicio en el que son atendidos ${ }^{15,21}$.

Otro de los instrumentos que facilitan conocer el grado de satisfacción, concretamente en hemodiálisis, es la Escala para la Evaluación de la Satisfacción del Paciente con el Servicio de Hemodiálisis (ESUR-HD), la cual atiende a 9 dominios de la dimensión de la satisfacción ${ }^{16}$.

Por último, bajo un enfoque de cumplimiento terapéutico, destaca el cuestionario de valoración SMAQ (Simplified Medication Adherence Questionnaire), siendo éste validado en la población española en pacientes con tratamiento renal sustitutivo. Dicho cuestionario consta de 6 preguntas de carácter dicotómico (SI/NO), por lo que cualquier respuesta en el sentido de no adherente implicará que al paciente se le considere no adherente al tratamiento prescrito ${ }^{22}$.
Interacción con enfermería. Aspectos más valorados Según diversos estudios, resulta evidente el crucial papel desempeñado por el profesional enfermero en el cuidado, seguridad y recuperación del paciente, en especial en aquellas entidades patológicas de carácter crónico ${ }^{7,10,12}$, conllevando consigo, en algunos casos, tratamientos sustitutorios o paliativos con un marcado carácter permanente, como es la díalisis ${ }^{17}$. En este sentido, las funciones inherentes de Enfermería llevadas a cabo en el paciente renal, como son el adecuado tratamiento, educación sanitaria o apoyo psicológico, permiten alcanzar unos estándares de calidad en los cuidados adecuados ${ }^{3,12,13}$ que, siendo enfocados sobre el contexto de la satisfacción, actúan sobre cada uno de los factores determinantes del mismo, intentando desarrollar los más altos niveles de satisfacción ${ }^{18}$. De ahí, que la mayoría de autores pongan énfasis en la necesidad de una adecuada educación sanitaria, por parte del profesional facultativo y enfermero, del paciente y familia respecto al proceso clínico y las características asociadas al mismo, que repercutirá directamente en el paciente ${ }^{21}$.

Finalmente, resulta evidente destacar aquellos atributos que mejor valoración han reportado por parte del paciente en diálisis, conformándose éstos como factores influyentes en el aumento del nivel de satisfacción percibida, destacando: amabilidad, disposición e interés, preparación técnica profesional, trato personalizado, confianza y seguridad clínica 3,7,13,23.

\section{Áreas de mejora}

La mayoría de estudios encontrados proponen como áreas de mejora ante la satisfacción del paciente: charlas dirigidas a pacientes y familiares en temas relacionados con información general y específica respecto a su proceso de enfermedad renal crónica ${ }^{10}$, así como el aporte de información requerida que permita aumentar el nivel de formación de profesionales sanitarios para asistir adecuadamente a este colectivo de pacientes ${ }^{7,13}$. En éste último, otro de los aspectos que precisan mejora es la correcta información sobre avances tecnológicos y monitores utilizados en el adecuado tratamiento de la ERCA $^{10,11}$.

Cabe hacer especial hincapié en la información referente a la medicación y a la dieta, puesto que resulta un factor determinante e influyente en la percepción de la propia salud del paciente, condicionando, en gran medida, los resultados obtenidos 3,12 .

Algunos artículos apuntan la necesidad de ampliación de las unidades de diálisis y, con ello, el aumento del 
personal que les atiende, conllevando consigo la reducción de tiempos de espera. Otro aspecto a mejorar es la calidad y cantidad de comida que se les facilita durante su estancia, siendo secundada por la necesidad mejora del transporte evitando esperas prolongadas $^{7,13}$.

\section{Discusión}

La ERCA, caracterizada por su evolución progresiva, lleva consigo un tratamiento prolongado y paliativo ante el fracaso renal objetivado, requiriendo al final de la última etapa de la enfermedad una TRS, como el trasplante rena|l1,12 0 alguna de las modalidades terapéuticas que conforman la diálisis ${ }^{17}$, la HD o la DP, en las que los pacientes precisan de unas necesidades especiales que repercuten en su estado bio-psicosocial $^{23}$, siendo éstas principalmente determinadas por la propia evolución clínica y el manejo terapéutico llevado a cabo aplicado a los mismos ${ }^{7,12}$.

En este sentido, la correcta valoración del nivel de satisfacción, así como las diferentes variables que la conforman ${ }^{7,14}$, se articulan como cruciales a la hora de conocer el grado de atención requerida por parte de los pacientes en diálisis, estableciéndose la estrategia terapéutica que mayor se adapte a las expectativas y necesidades individuales alteradas de cada paciente $\mathrm{e}^{7,11,16,17,23}$. Este hecho no resulta aislado, ya que el nivel de satisfacción permite establecer planes de mejora, aumentando la calidad de la atención y cuidados llevados a cabo por parte del personal sanitario implicado en el proceso de tratamiento en diálisis ${ }^{10.11}$.

Actualmente, los profesionales implicados en la atención del paciente renal tienen a su disposición un abanico de herramientas de valoración que permiten conocer, objetivamente, multitud de aspectos de interés relacionados con el propio proceso clínico y la esfera psicosocial de este tipo de pacientes ${ }^{23,24}$.

Sin embargo, no existen cuestionarios específicos que midan la satisfacción del paciente en diálisis, y los existentes no han sido adaptados a la población española en diálisis. Los principales cuestionarios de valoración de esta variable encontrados en esta revisión, evalúan los diferentes factores asociados a la satisfacción del paciente en diálisis, destacando, entre otros: las escalas tipo Likert $^{18}$ y los cuestionarios CARE-Q ${ }^{19}$, LOPSS $12^{20}$ y SUR-HD ${ }^{16}$ para conocer el nivel de satisfacción de pacientes nefrológicos, así como el cuestionario
SERVQHOS $3,7,10-13$ para valorar el nivel de calidad de vida del paciente en diálisis. Según López MC et al. ${ }^{13}$, la utilidad derivada del uso de dichos instrumentos de valoración radica en la fiabilidad y precisión de los mismos, requiriendo de nuevos estudios que formulen nuevas herramientas que permitan atender a las recientes necesidades originadas en el paciente renal, traducidos en términos de calidad y satisfacción ${ }^{17,21}$.

Por otro lado, según los resultados obtenidos, destaca el alto grado de satisfacción del paciente en diálisis, en general, y mejor percepción de la mismas de los pacientes en DP respecto a los de HD ${ }^{14}$. Este hecho es asociado a determinantes como la calidad de vida ${ }^{15,16}$, funcionamiento social ${ }^{15}$ y seguridad clínica ${ }^{7,11,13}$. Sin embargo, los factores que mayor grado de insatisfacción han sido reportados son principalmente, incorrecta información acerca del régimen terapéutico y dieta ${ }^{3,7,10-13}$, así como falta formación y número de personal adecuado en la unidad ${ }^{7,10,13}$. Reid $C$ et al. $^{23} \mathrm{y}$ Ruíz B et al. ${ }^{24}$, establecen que las alteraciones de los roles socio-familiares, así como del nivel de funcionamiento del paciente renal, suponen un riesgo añadido al grado de satisfacción, pudiendo afectar ciertas áreas de valoración que actualmente se encuentran bien conservadas.

Atendiendo al nivel de calidad percibida por parte de los usuarios de diálisis ante los cuidados de enfermería, la satisfacción reportada es elevada, coincidiendo con la mayor parte de producción científica disponible $7,11-13,24$. Las acciones derivadas de la educación sanitaria llevada a cabo, así como el establecimiento de un adecuado tratamiento y apoyo psicológico ejercido durante el proceso de atención terapéutica por parte del profesional enfermero, reportan los mayores resultados positivos de satisfacción de este grupo de pacientes $^{17,21,22,24}$.

\section{Limitaciones del estudio}

Como principales limitaciones de nuestro estudio cabe destacar los pocos estudios localizados y la variabilidad existente en la metodología e instrumentos de medida utilizados para evaluar la satisfacción, y por tanto, las diferentes dimensiones utilizadas en cada uno de ellos no permiten agrupar resultados. También en los artículos seleccionados, no existe homogeneidad respecto a la técnica de diálisis en la que se ha evaluado la satisfacción (HD, DP -intermitente ambulatoria, intermitente diurna, tidal etc..-). 


\section{Consideraciones prácticas}

Como consideraciones prácticas de la presente revisión, cabe destacar la importancia de llevar a cabo este tipo de estudios en la práctica clínica de forma periódica, estableciendo como principal objetivo conocer las expectativas de los pacientes, así como el establecimiento de puntos de mejora y fortalecimiento de aspectos clave en la satisfacción de los usuarios de diálisis, siendo éstos últimos partícipes en el reporte de opiniones y sugerencias relevantes para la mejora continua del proceso asistencial. Del mismo modo, resulta evidente la necesidad de difusión de los resultados obtenidos hacia el profesional sanitario, sirviendo como incentivo en el puesto profesional desempeñado.

A la vista de estos resultados, podemos afirmar que:

-Los factores más influyentes en la satisfacción del paciente en diálisis son, principalmente, la confianza transmitida y la disposición del personal sanitario en la prestación de ayuda, el trato personalizado, la amabilidad y seguridad transmitida, la destreza para pinchar la fístula, el interés por la resolución de problemas asociados, así como la información relacionada con la higiene y la rapidez de respuesta requerida. Al contrario, los factores menos satisfactorios son la escasa capacitación del personal, la información médica, la tecnología de los equipos, el tiempo de espera de atención médica, así como la información referente a la dieta y medicación prescrita.

-Respecto a los instrumentos de medida, hay que indicar que no existe un instrumento de medida específico, para evaluar la satisfacción del paciente en diálisis; y los existentes no están adaptados a la población española en diálisis.

-En cuanto a los atributos que los pacientes en diálisis perciben como más satisfactorios en su relación con enfermería, destacan la amabilidad, disposición e interés, preparación técnica profesional, trato personalizado, confianza y seguridad clínica.

Finalmente, es importante indicar la necesidad de evaluar periódicamente el grado de satisfacción del paciente en diálisis, para no solo responder a sus necesidades sino también a sus expectativas y detección de áreas de mejora asistenciales. Este hecho resulta fundamental en la conformación y diseño de planes de gestión y atención sanitaria, conllevando una adecuada actuación clínica, así como el establecimiento de la seguridad clí- nica pertinente, siguiendo los más altos estándares de calidad.

\section{Conflictos de interés}

Los autores declaran no tener conflictos de interés potenciales relacionados con los contenidos del presente artículo.

Recibido: 28-12-18

Revisado: 15-01-19

Modificado: 30-01-19

Aceptado: 15-02-19

\section{Bibliografía}

1. Pérez T, Rodríguez A, Suárez J, Rodríguez L, García MA, Rodríguez Pérez JC. Satisfacción del paciente en una Unidad de Diálisis. ¿Qué factores modulan la satisfacción del paciente en diálisis? Enferm Nefrol. 2012;15(2):101-7. D0I: 10.4321/S225428842012000200004.

2. Miguel $M$, Valdés $C$, Rábano $M$, Artos $Y$, Cabello $P$, De Castro $\mathrm{N}$ et al. Variables asociadas a la satisfacción del paciente en una unidad de hemodiálisis. Rev Soc Esp Enferm Nefrol. 2009;12(1):19-25. [Consultado 13 abril 2018]. Disponible en: scielo.isciii. es/pdf/nefro/v12nl/originall3.pdf

3. García R, Fernández J, González I, Fernández C, Fernández M, Díaz C. Satisfacción del paciente en diálisis (Hemodiálisis y Diálisis Peritoneal): aspectos a mejorar. Enferm Nefrol (Mad). 2016;19(3):24854. DOI: $10.4321 / S 2254-28842016000300007$.

4. Largo CM, Zamaniego MA. Calidad y nivel de satisfacción en la Atención de los pacientes del centro de DIALILIFE-CUENCA 2017. Universidad de Cuenca. 2018. [Consultado 23 marzo 2018]. Disponible en: http://dspace.ucuenca.edu.ec/handle/123456789/30385. 
5. Ocaña J, Montero D. Calidad de vida en pacientes enfermos renales crónicos sometidos a diálisis peritoneal ambulatoria versus hemodiálisis, en el hospital Carlos Andrade Marín Quito período Junio-Septiembre 2013. Universidad Nacional de Chimborazo. 2013. [Consultado 17 marzo 2018]. Disponible en: http://dspace.unach.edu.ec/handle/51000/105.

6. Hernández $M E$, Ochando $A$, Lorenzo $S$, Orbes $P$, López K. Factores determinantes de la satisfacción del paciente en tratamiento renal sustitutivo. Rev Soc Esp Enferm Nefrol. 2007;10(1):6-13. DOI: $10.4321 / s 1139-13752007000100002$.

7. Sanz M, Garrido L, Caro C. Factores que influyen en la satisfacción del paciente de diálisis con enfermería. Enferm Nefrol. 2017;20(1):66-75. DOI: $10.4321 /$ S2254-28842017000100009.

8. Castro $E$, Cadena G. Dimensiones de la satisfacción del paciente con insuficiencia renal crónica respecto a la calidad del cuidado de enfermería. Rev Enferm Mex Seguro Soc. 2017;25(4):271-8. [Consultado 5 mayo 2018]. Disponible en: www.medigraphic.com/ pdfs/enfermeriaimss/eim-2017/eim174e.pfd.

9. Guirao JA. Utilidad y tipos de revisión de literatura. Ene [Internet]. 2015;9(2). [Consultado 23 marzo 2018]. Disponible en: http://scielo.isciii.es/scielo. php?script=sci_arttext\&pid=S1988-.

10. Rodríguez D, Sanjuán D. Satisfacción percibida por los pacientes en el servicio de hemodiálisis del hospital general de La Palma. Enferm Nefrol (Mad). 2013;16(2):75-82. DOI: 10.4321/ S225428842013000200002.

11. Martín RA. Satisfacción del paciente en HD como criterio de calidad asistencial en enfermería. Rev Mex Urol. 2014;74(5):292-5. D0I: https://doi.org/10.1016/j.uromx.2014.09.008.

12. Gómez $M$, Moreno $P$, Miralles $F$, Sánchez MD, Sánchez MA, Pérez G. Cuidados de calidad y satisfacción del paciente con enfermería en hemodiálisis. Enferm Nefrol (Mad).2014;17(Supl 1)S88-108.

13. López MC, Luque I, Gómez VE, Casas R, Crespo R. La satisfacción del paciente como componente de la calidad en las unidades de diálisis. Enferm Nefrol (Mad).2016;19(2):125-33. D0I: 10.4321 / S225428842016000200004.
14. Rebollo A, Pons ME, Macías MJ, Cabrera S. Valoración de la acogida de pacientes que inician terapia renal sustitutiva: Nivel de satisfacción. Enferm Nefrol. 2015;18(2):97-102. DOI: 10.4321/S225428842015000200004.

15. Aguilar F, Fernandes I, Camargo JM, Bucaneve J, Prado NM, Terumy $C$ et al. Quality of life in chronic renal patients on hemodialysis or peritoneal dialysis: a comparative study in a referral service. $\mathrm{J}$ Bras Nefrol. 2015 (octubre-diciembre);37(4):467-74. DOI: 10.5935 / 0101-2800.20150074.

16. Sanabria M, Tobón J, Certuche MC, Sánchez R. Validation of an instrument for measuring satisfaction of patients undergoing hemodialysis. BMC Health Serv Res. 2017;17:321. D0I: 10.1186 / s12913017-2251-y.

17. Ángel ZE, Duque GA, Tovar DL. Cuidados de enfermería en el paciente con enfermedad renal crónica en hemodiálisis: una revisión sistemática. Enferm Nefrol. 2016;19(3):202-13. DOI: 10.4321/S2254 28842016000300003.

18. Rocha MR, Vega J, Reséndiz G, López G. Determinación de la calidad a través de la satisfacción del paciente y atención de enfermería en clínica ISSSTE, Rioverde, México. Rev. Paraninfo Digital. 2014;21. [Consultado 13 abril 2018]. Disponible en: http://www.index-f.com/para/n21/034.php.

19. Urbina MA, Ávila $H$, Gutiérrez G, Vázquez L, Martínez ML, Hernández MA. Satisfacción del cuidado enfermero en pacientes con Enfermedad Renal Crónica. Rev. Paraninfo Digital. 2016;25. [Consultado 17 marzo 2018]. Disponible en: http://www.index-f. com/para/n25/021.php.

20. Morga S, Navarro MA, Torralba MJ, Saura AM. Un estudio de calidad de vida y grado de satisfacción con los cuidados de enfermería en pacientes con insuficiencia renal crónica. Rev. Paraninfo Digital. 2014; 20. [Consultado 17 marzo 2018]. Disponible en: http://www.index-f.com/para/n20/232.php.

21. Palmer SC, de Berardis G, Craig JC, Tong A, ToneIli $M$, Pellegrini $F$ et al. Patient satisfaction with in-centre haemodialysis care: an international survey. BMJ Open. 2014;4:e005020. D0I: 10.1136/ bmjopen-2014-005020. 
22. Huertas $M P$, Pérez $R$, Albalate $M$, De Sequera $P$, Ortega $M$, Puerta $M$, et al. Factores psicosociales y adherencia al tratamiento farmacológico en pacientes en hemodiálisis crónica. Rev. Nefrol. 2014; 34(6):737-42. DOI: 10.3265/Nefrologia.pre2014. Jul.12477.

23. Reid C, Seymour J, Jones C. A Thematic Synthesis of the Experiences of Adults Living with Hemodialysis. Clin J Am Soc Nephrol. 2016 (Jul);11:1206-18. D0I: 10.2215 / CJN.10561015.

24. Ruiz de Alegría B, Basabe N, Saracho R. El afrontamiento como predictor de la calidad de vida en diálisis: un estudio longitudinal y multicéntrico. Rev. Nefrol. 2013;33(3):342-54. DOI: 10.3265/Nefrologia.pre2013.Feb.11771.

Este artículo se distribuye bajo una Licencia Creative Commons Atribución-NoComercial 4.0 Internacional. https://creativecommons.org/licenses/by-nc/4.0/

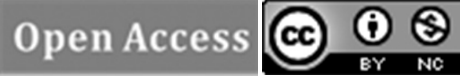

